\title{
Produção de metano entérico em pastagens tropicais ${ }^{1}$
}

\author{
Enteric Methane production in tropical pastures
}

\author{
BERCHIELLI, Telma Teresinha" ${ }^{2 *}$ MESSANA, Juliana Duarte ${ }^{2}$; CANESIN, Roberta \\ Carrilho $^{2}$
}

\author{
${ }^{1}$ Revisão apresentada no Congresso Brasileiro de Zootecnia (ZOOTEC 2011). \\ ${ }^{2}$ Universidade Estadual de São Paulo, Faculdade de Ciências Agrárias e Veterinárias, Departamento de \\ Zootecnia, Jaboticabal, São Paulo, Brasil. \\ *Endereço para correspondência: ttberchi@fcav.unesp.br
}

\section{RESUMO}

$\mathrm{Na}$ presente revisão, buscou-se apresentar os principais impactos ambientais causados pela pecuária, sobretudo, em relação às emissões de gases efeito estufa (GEE). Além disso, buscou-se apresentar possíveis formas de mitigar essas externalidades. A criação de bovinos, no Brasil, acontece de forma extensiva, muitas vezes em áreas com pastagem degradada e, portanto, de baixa produtividade. Isso possibilita à atividade uma oportunidade de redução do impacto causado ao meio ambiente, uma vez que ações tomadas, no sentido de melhorar o rendimento animal, devem resultar em um menor consumo de recursos naturais (terra e água) e maior eficiência do sistema digestivo animal. Os principais problemas apontados pelos pesquisadores, no que tange à pecuária extensiva, são o metano emitido pela fermentação entérica dos ruminantes, o óxido nitroso emitido pelos dejetos dos animais em pastejo e o dióxido de carbono trocado pelo solo e vegetação. Muitos fatores influenciam a produção de $\mathrm{CH}_{4}$ entérico dos ruminantes, inclusive o tipo de carboidrato fermentado, o sistema digestivo do animal, a quantidade e o tipo de alimentos consumidos. Diante do exposto, pesquisadores têm desenvolvido tecnologias para reduzir a emissão de metano, através da melhoria das práticas de manejo alimentar, manipulação ruminal, por meio de suplementação com monensina, lipídios, ácidos orgânicos e compostos de plantas. Outras estratégias de redução de metano que foram investigadas são: defaunação e vacinas, que buscam inibir micro-organismos metanogênicos e a metanogênese. Assim, a busca por sistemas de produção eficientes tem sido uma das perspectivas da pecuária mundial para reduzir a emissão de poluentes e intensificar a produção animal.

Palavras-chave: aquecimento global, manipulação ruminal, mitigação de GEE, ruminantes.

\section{SUMMARY}

This review aimed to present the main environmental impacts related to livestock, especially as regards the emissions of greenhouse gases (GHGs) and the possible ways to mitigate these externalities. The creation of ruminants in Brazil comes mainly extensively, often in areas of degraded pasture and hence low productivity. This fact enables the activity a great opportunity to reduce the impact to the environment, since actions taken to improve animal performance should result in a lower consumption of natural resources (land and water) and greater efficiency of the digestive system animal. The main problems identified by researchers in relation to cattle production are the methane emitted from enteric fermentation of ruminants, nitrous oxide emitted by feces of grazing animals and carbon dioxide exchanged by soil and vegetation. Many factors influence the production of $\mathrm{CH}_{4}$ from enteric ruminants, including the type of carbohydrate fermentation, the animal's digestive system, and the amount and type of food consumed. In this light, researchers have been working technologies to reduce methane emissions through improved food handling practices, manipulation of rumen by supplementation with monensin, lipids, organic acids and plant compounds. Other strategies to reduce methane that were investigated are defaunation and vaccines, which seek to inhibit methanogens and methanogenesis. Thus, the search for more efficient production systems has been one of the prospects of livestock worldwide, aiming to reduce emissions and increase animal production.

Keywords: global warming, mitigation, rumen manipulation, ruminants. 


\section{INTRODUÇÃOO}

As principais fontes de emissão de gases de efeito estufa (GEE) do Brasil, estão concentradas em dois setores: o primeiro, uso do solo, mudança desse uso e de florestas, que contribuem com 55\%; o segundo é a agropecuária, responsável por outros $25 \%$ da emissão total (BRASIL, 2004). Grande parte dessa remessa é creditada à pecuária, por expelir $\mathrm{CH}_{4}$, oriundo da fermentação entérica dos ruminantes, e por emitir $\mathrm{N}_{2} \mathrm{O}$, decorrente da deposição de dejetos dos animais, que compreendem $68 \%$ e $43 \%$ das emissões totais desses gases no país. Além disso, o lançamento do $\mathrm{CO}_{2}$ para atmosfera está relacionado à conversão de florestas para aqueles fins. Em áreas de pastagens, portanto, ocorrem trocas de gases com força radioativa, intimamente ligadas às práticas de manejo.

Tal fato está atrelado à posição de destaque do país em relação à produção mundial de carne, já que possui o segundo maior rebanho de gado do mundo, com 205 milhões de cabeças, o que faz da pecuária uma atividade de grande importância econômica para o país (IBGE, 2010). A pecuária nacional é baseada em pastagens, principalmente, do gênero Brachiaria, que ocupa cerca de $50 \%$ da área de pastagens cultivadas, devido ao baixo custo de produção da alimentação de rebanhos, se comparado aos sistemas que utilizam animais confinados e grãos na dieta.. Incertezas, em relação à emissão de gases de efeito estufa pela pecuária, apontam a possibilidade de mitigação desses gases, que pode melhorar o cenário do ramo pecuarista no ranking das emissões brasileiras.

Os ruminantes representam uma das poucas fontes produtoras de $\mathrm{CH}_{4}$ que podem ser manipuladas, pois a produção de metano por bovinos é proveniente da fermentação ruminal, que está relacionada ao tipo de animal, ao consumo e à digestibilidade de alimento (RIVERA et al., 2010). Assim, é possível reduzir a produção desse gás pela modificação da fermentação ruminal, obtida por alteração do volumoso, do tipo e da quantidade de carboidrato suplementado à dieta, pela adição de lipídios e pela manipulação da microbiota ruminal com aditivos alimentares (MOHAMMED et al., 2004; SHIBATA \& TERADA, 2010 ).

Dessa forma, a busca por sistemas de produção eficientes, que reduzam a emissão de gases de efeito estufa por unidade de produto, tem sido uma das perspectivas da pecuária mundial. Essas perspectivas estão relacionadas, convém destacar, ao melhor aproveitamento do alimento e à redução da idade de abate (BEAUCHEMIN et al., 2008).

\section{ATUAÇÃO DOS RUMINANTES NO AQUECIMENTO GLOBAL}

O rebanho bovino do Brasil é estimado em cerca de 205 milhões de cabeças (IBGE, 2010), em contínuo crescimento e com avanços nos índices de produtividade. Diante desses números, a pecuária tem sido apontada como uma das atividades que mais prejudicam o meio ambiente. Esta contribui negativamente com emissões de metano por meio da fermentação entérica e do manejo de dejetos animais.

A fermentação do alimento ingerido, no rúmen, é um processo anaeróbio efetuado pela população microbiana ruminal, em que os carboidratos celulósicos são convertidos em ácidos graxos de cadeia curta, os quais são utilizados pelo animal como fonte de energia. Bactérias metanogênicas, 
presentes no rúmen, obtêm energia para seu crescimento ao utilizar $\mathrm{H}_{2}$ para reduzir $\mathrm{CO}_{2}$ e formar metano $\left(\mathrm{CH}_{4}\right)$, o qual é eructado ou exalado para a atmosfera (COTTLE et al., 2011).

De acordo com o USEPA (2000), a emissão global de metano de origem entérica é estimada em 80 milhões de toneladas ao ano, o que corresponde a cerca de $22 \%$ das emissões totais de metano gerada por fontes antrópicas, de modo a representar $3,3 \%$ do total dos gases de efeito estufa. $O$ metano aumenta, a cada ano, numa taxa de $0,9 \%$ na atmosfera, mas a grande quantidade de gases é representada pelo $\mathrm{CO}_{2}$ com $60 \%$ do total.

Acredita-se que as emissões provenientes da agricultura constituem uma pequena parte das emissões totais de gases de efeito estufa (SHIBATA \& TERADA, 2010). Segundo o MCT (2010), em 2005, o total de emissões de metano pela pecuária brasileira foi estimado em $12.210 \mathrm{Gg}$, do qual 11.487 Gg podem ser atribuídas à fermentação entérica e 723 Gg a sistemas de manejo de dejetos animais. Em 2005, as categorias de gado bovino contribuíram com $96,9 \%$ das emissões de metano por fermentação entérica e $91,1 \%$ das emissões totais de metano da pecuária.

$\mathrm{O}$ aumento da produção de gases poluentes é impulsionado pelo avanço da bovinocultura brasileira, que tem gerado um grande entrave, isto é, a mais nova barreira imposta à pecuária brasileira conhecida como o "boi do metano", (PINEDA, 2010). Informações banalizadas pela mídia contribuem ainda para acentuar falhas de comunicação entre os diversos setores da cadeia produtiva, de modo a tornar a carne bovina a grande vilã do desenvolvimento sustentável.

Inúmeras publicações falam sobre a quantidade de metano emitida pelos bovinos; afirmam que a cadeia da carne é um perigo para o ambiente, condenam o consumo da carne como alimento. Grupos minoritários se utilizam dos meios de comunicação para fazer propaganda, e até algumas autoridades fazem coro, na maioria dos casos, sem fundamentos técnicos. Nesse sentido, por exemplo, passearam em Copenhague teorias construídas à luz de um obscurantismo medieval (PINEDA, 2010).

Outro ponto que vilipende ainda mais a imagem da pecuária nacional, refere-se ao recente crescimento da devastação de florestas na Amazônia, pois são atribuídos à pecuária o desmatamento $\mathrm{e}$ as queimadas. Sob todos os aspectos, o prejuízo causado pelo desmatamento adquire enormes proporções, e a rastreabilidade do gado será utilizada para que os países importadores rejeitem a carne produzida na região Amazônica (FELÍCIO, 2008).

Apesar de grande emissora, a pecuária mostra ter um grande potencial de sequestro de carbono, através de pastagens bem manejadas. A emissão nacional é um pouco maior que $1 \mathrm{Mg}$ $\mathrm{CO}_{2}$ eq/ha, enquanto o sequestro pode atingir $0,78 \mathrm{Mg} \mathrm{CO}$ eq/ha (ZEN et al., 2008). Assim, o investimento em pastagem poderia aumentar eficiência produtiva animal e, assim, reduzir a quantidade de GEE emitidos por quilo de carne produzida.

\section{ESTRATÉGIAS DA REDUÇÃO DA PRODUÇÃO DE METANO POR RUMINANTES}

A produção de metano entérico é parte do processo digestivo dos herbívoros ruminantes e ocorre no rúmen. A intensidade da emissão de metano, proveniente da fermentação ruminal, depende principalmente do tipo de 
animal, do consumo de alimentos e do grau de digestibilidade da massa ingerida. Segundo Cottle et al. (2011), as indicações para reduzi as emissões de metano pela pecuária estão ligadas à melhoria da dieta, à melhoria de pastagens, à suplementação alimentar, ao aumento da capacidade produtiva dos animais e à outras medidas que refletem na melhor eficiência produtiva e resultam em ciclos de produção mais curtos.

Bovinos criados em ambiente tropical, mantidos em sistemas de pastejo, apresentam a produção de metano entérico afetada pela constituição morfológica e composição química das plantas forrageiras desse ambiente. Além disso, a temperatura ambiental também pode afetar a produção de gás, tanto indiretamente pela interferência na composição química das plantas, como de forma direta, com alterações no comportamento ingestivo do animal e nas características da digestão (HOOK et al., 2010).

Segundo Pedreira et al. (2009), a relação parede celular:conteúdo celular e a constituição da parede celular das plantas forrageiras são os principais fatores envolvidos na produção de metano. Nesse sentido, existe um comportamento diferente entre plantas forrageiras de clima tropical $\left(\mathrm{C}_{4}\right)$ e de clima temperado $\left(\mathrm{C}_{3}\right)$. As características das gramíneas $\mathrm{C}_{4}$ podem conduzir a diferentes interpretações quanto ao potencial de fornecimento de substrato para fermentações que geram metano no rúmen. Estas forrageiras, por possuírem maiores proporções de fibra que as plantas de metabolismo $\mathrm{C}_{3}$ (NELSON \& MOSER, 1994), favorecem a fermentação acética, com maior produção de metano (g/dia). Por outro lado, essa fibra apresenta baixa digestibilidade e menor velocidade de fermentação, quando comparadas às plantas de clima temperado, logo, fornecem menor quantidade de substrato para os micro-organismos metanogênicos.

Segundo Cottle et al. (2011), nas forragens de baixa qualidade, a adição de nutrientes para os micro-organismos incrementa a eficiência do crescimento microbiano, pois aumenta a eficiência do processo fermentativo no rúmen, com decréscimo nas metanogênicas, por unidade de carboidratos degradados. Ocorre também aumento na produção de metano por animal, pois, uma quantidade maior de matéria orgânica é fermentada.

Se por um lado, a produção de metano no rúmen caracteriza uma ineficiência no processo digestivo dos ruminantes, por outro lado, se faz necessária. $\mathrm{O}$ acúmulo de $\mathrm{H}^{+}$inibe os sistemas enzimáticos, principalmente os processos que envolvem a nicotinamida adenosina difosfato (NADH $+\mathrm{H}^{+} \leftrightarrow \mathrm{NAD}^{+}$) (MCALLISTER \& NEWBOLD, 2008) e a regulação do $\mathrm{pH}$ ruminal. Dessa forma, há necessidade de drenar as moléculas de $\mathrm{H}_{2}$ produzidas para um ótimo funcionamento do rúmen, e o principal meio é a produção de metano.

As pesquisas sugerem que o passo inicial, na tentativa de reduzir a participação da bovinocultura na mudança climática global, seja o aumento da produtividade, através do fornecimento de alimentos de melhor qualidade, o que, segundo pesquisadores, poderia diminuir $10 \%$ da emissão de metano por quilo de carne produzida (ZEN et al., 2008). Assim, é imprescindível a intensificação dos sistemas de produção de diversas maneiras. Dentre as possíveis, destacam-se: o desenvolvimento de especialidades nas diferentes fases até a produção de carne, melhoria de pastagens, suplementação alimentar e implantação do sistema rotativo; 
semiconfinamento e confinamento; e sistemas alternativos como a integração lavoura-pecuária e sistemas silvipastoris.

Segundo Barioni et al. (2007), se a eficiência produtiva continuar em aumento conforme as mesmas proporções dos últimos 15 anos, é provável que em 2025 a produção de bovinos seja $25 \%$ maior, com os níveis de emissão de GEE apenas 3\% maiores, com uma redução de $18 \%$ na relação $\mathrm{kg} \mathrm{CH} / \mathrm{kg}$ de carne produzida.

Portanto, torna-se viável o uso de animais de alto potencial genético para maximizar o ganho de peso, obter maior rendimento de carcaça e precocidade de abate. Evidentemente, os objetivos das atuações zootécnicas, que resultam em intensificação dos sistemas de produção, é reduzir a remessa de metano por kg de peso vivo através do aumento do ganho de peso diário dos animais. Um animal, que se desenvolve mais rápido, atingirá o peso de abate em uma idade mais jovem. Nessa hipótese, menos alimento é utilizado na manutenção do animal, e as emissões totais de metano, ao longo do ciclo de vida, também serão reduzidas, o que resulta em menos $\mathrm{CH}_{4}$ por $\mathrm{kg}$ de peso vivo ou por $\mathrm{kg}$ de carne bovina produzida. Devido à diminuição das exigências de manutenção, o $\mathrm{CH}_{4}$ emitido por peso final em $\mathrm{kg}$ cai rapidamente, em termos absolutos e relativos, quando comparados à idade no abate.

Canesin (2009) verificou que a produção de metano de bovinos Nelore, mantidos em pastagem de Brachiaria brizantha cv. Marandu, submetidos a diferentes frequências de suplementação, foram de $7,4 \mathrm{~g} / \mathrm{h}$ e $176,8 \mathrm{~g} /$ dia no mês de setembro, e no mês de novembro $13,0 \mathrm{~g} / \mathrm{h}$ e $311,0 \mathrm{~g} / \mathrm{dia}$ (Tabela 1). Primavesi et al. (2004) obtiveram valores semelhantes de
$7,6 \mathrm{~g} / \mathrm{h}$ e 181,0g/dia de produção de metano entérico em novilhas mestiças leiteiras brasileiras mantidas em pastagem de capim-Marandu, sem adubação, e em vacas secas mantidas em pastagem de capim tobiatã, adubado no verão, foram de $12,3 \mathrm{~g} / \mathrm{h}$ e $295,0 \mathrm{~g} / \mathrm{dia}$ para o mês de setembro e novembro, respectivamente (Tabela 2).

$\mathrm{O}$ percentual de $\mathrm{CH}_{4}$, perdido a partir da energia bruta ingerida, é estimado entre $5,5 \%$ e $6,5 \%$ (USEPA, 2000) para as estimativas na América do Norte e leste Europeu. No entanto, Canesin (2009) verificou uma perda de energia na forma de metano $\left(\mathrm{CH}_{4} \% \mathrm{EBi}\right)$ de $8,5 \%$ e $12,3 \%$ nos meses de setembro e novembro, respectivamente.

Foram observadas por Kurihara et al. (1999), em bovinos alimentados com gramíneas de clima tropical, perdas de energia de 10,4\% com capim-rodes (Chloris gayana) e 11,4\% com capimengleton (Dicanthium aristatum). Altas perdas de energia na forma de metano também foram verificadas por Primavesi et al. (2004) em fêmeas mestiças, cujos valores médios de 10,6 e $9,1 \%$ em vacas lactantes e secas, respectivamente, em pastagem adubada e, de 9,6 e 7,8\% em novilhas mantidas em pastagem adubadas e sem adubação, respectivamente. Em função dos resultados obtidos, as perdas de 6,5 a $7,5 \%$, previstas por IPCC (2006) estão abaixo das emissões de $\mathrm{CH}_{4}$ encontradas por bovinos em condições tropicais.

Pedreira et al. (2009) observaram que a produção de metano variou em função da categoria de animal e sistemas de produção (Tabela 3). Os autores verificaram que animais da raça holandesa produziram mais metano (299,3g/dia) que as mestiças (264,2g/dia). Observaram também que vacas secas e novilhas produziram menos metano (268,8 e 222,6g/dia, respectivamente) 
que vacas em lactação (353,8g/dia), e animais da raça holandesa, com maior potencial de produção de leite, perderam menos $\mathrm{CH}_{4}$ por unidade de matéria seca ingerida $(19,1 \mathrm{~g} / \mathrm{kg})$ que as mestiças $(22,0 \mathrm{~g} / \mathrm{kg})$. A maior produção de metano foi observada nas novilhas mantidas em pastagens adubadas (sistema intensivo) de 222,6g/dia, resultado maior do que o encontrado quando animais dessa categoria se encontravam em pastagens não adubadas (179,2g/dia).

Tabela 1. Produção de metano, expressos de diversas formas, em bovinos mantidos em pastagem em função das frequências de suplementação e meses avaliados

\begin{tabular}{|c|c|c|c|c|c|c|c|}
\hline \multirow{2}{*}{ Item } & \multicolumn{3}{|c|}{ Frequência de suplementação } & \multirow{2}{*}{ Média } & \multicolumn{3}{|c|}{$P$} \\
\hline & SD & SS & DA & & $\mathrm{F}$ & M & $\mathrm{F}^{*} \mathrm{M}$ \\
\hline \multicolumn{8}{|c|}{$\mathrm{CH}_{4}(\mathrm{~g} / \mathrm{h})$} \\
\hline Setembro & 6,8 & 7,4 & 7,9 & $7,4^{\mathrm{B}}$ & 0,8107 & 0,0041 & 0,9568 \\
\hline Novembro & 12,0 & 13,7 & 13,2 & $13,0 \mathrm{~A}$ & - & - & - \\
\hline \multicolumn{8}{|c|}{$\mathrm{CH}_{4}$ (g/dia) } \\
\hline Setembro & 163,6 & 178,0 & 188,9 & $176,8^{\mathrm{B}}$ & 0,8107 & 0,0041 & 0,9568 \\
\hline Novembro & 288,3 & 328,5 & 316,1 & $311,0^{\mathrm{A}}$ & - & - & - \\
\hline \multicolumn{8}{|c|}{$\mathrm{CH}_{4}\left(\mathrm{~g} / \mathrm{d} / \mathrm{kg}^{0,75}\right)$} \\
\hline Setembro & 1,9 & 2,1 & 2,3 & $2,1^{\mathrm{B}}$ & 0,3783 & 0,0073 & 0,6407 \\
\hline Novembro & 3,0 & 2,6 & 3,3 & $3,0^{\mathrm{A}}$ & - & - & - \\
\hline \multicolumn{8}{|c|}{$\mathrm{CH}_{4}(\mathrm{Mcal} / \mathrm{dia})$} \\
\hline Setembro & 2,2 & 2,4 & 2,5 & $2,3^{\mathrm{B}}$ & 0,4757 & 0,0013 & 0,5786 \\
\hline Novembro & 3,8 & 3,2 & 4,2 & $3,7^{\mathrm{A}}$ & - & - & - \\
\hline \multicolumn{8}{|c|}{$\mathrm{CH}_{4}(\% \mathrm{EBi})$} \\
\hline Setembro & 7,9 & 8,0 & 9,5 & $8,5^{\mathrm{B}}$ & 0,3771 & 0,0099 & 0,8909 \\
\hline Novembro & 12,1 & 11,0 & 14,0 & $12,3^{\mathrm{A}}$ & - & - & - \\
\hline \multicolumn{8}{|c|}{$\mathrm{CH}_{4}(\mathrm{~g} / \mathrm{kg} \mathrm{MSi})$} \\
\hline Setembro & 21,3 & 21,5 & 25,7 & $22,8^{\mathrm{B}}$ & 0,3822 & 0,0029 & 0,8792 \\
\hline Novembro & 35,6 & 32,3 & 41,1 & $36,3^{\mathrm{A}}$ & - & - & - \\
\hline \multicolumn{8}{|c|}{$\mathrm{CH}_{4}(\mathrm{~g} / \mathrm{kg} \mathrm{MOi})$} \\
\hline Setembro & 22,2 & 22,8 & 27,0 & $24,0^{\mathrm{B}}$ & 0,3555 & 0,0027 & 0,8300 \\
\hline Novembro & 37,6 & 33,5 & 43,4 & $38,2^{\mathrm{A}}$ & - & - & - \\
\hline \multicolumn{8}{|c|}{$\mathrm{CH}_{4}(\mathrm{~g} / \mathrm{kg} \mathrm{FDNi})$} \\
\hline Setembro & 40,5 & 39,7 & 48,4 & $42,9^{\mathrm{B}}$ & 0,4419 & 0,0015 & 0,9283 \\
\hline Novembro & 72,4 & 67,3 & 84,1 & $74,6^{\mathrm{A}}$ & - & - & - \\
\hline \multicolumn{8}{|c|}{$\mathrm{CH}_{4}(\mathrm{~g} / \mathrm{Mcal} \mathrm{EBi})$} \\
\hline Setembro & 6,0 & 6,1 & 7,2 & $6,4^{\mathrm{B}}$ & 0,3717 & 0,0103 & 0,8822 \\
\hline Novembro & 9,2 & 8,3 & 10,6 & $9,4^{\mathrm{A}}$ & - & - & - \\
\hline
\end{tabular}

Médias seguidas por diferentes letras maiúsculas nas colunas diferem entre si estatisticamente pelo teste de Tukey a 5\% de significância.

$\mathrm{SD}=$ suplementação diária; $\mathrm{SS}$ = suplementação oferecida de segunda à sexta-feira e suspensa aos sábados e domingo; $\mathrm{DA}=$ suplementação em dias alternados. \% $\mathrm{EBi}=$ metano em porcentagem da $\mathrm{EB}$ ingerida, $\mathrm{MSi}=$ matéria seca ingerida, $\mathrm{MOi}=$ matéria orgânica ingerida, $\mathrm{FDNi}=$ fibra em detergente neutro ingerido, $\mathrm{EBi}=$ energia bruta ingerida. $P=$ significância; $\mathrm{F}=$ frequência de suplementação; $\mathrm{M}=$ meses avaliados (CANESIN, 2009). 
Tabela 2. Emissão de metano $\left(\mathrm{CH}_{4}\right)$ por diferentes grupos genéticos e categorias de fêmeas bovinas leiteiras, em pastagens formadas com forrageiras tropicais, fertilizadas ou não

\begin{tabular}{|c|c|c|c|c|}
\hline \multirow{2}{*}{$\begin{array}{l}\text { Grupo genético e categoria } \\
\text { animal }\end{array}$} & \multicolumn{4}{|c|}{ Holandês Preto e Branco } \\
\hline & Vaca em lactação & Vaca não lactante & Novilha & Novilha \\
\hline & \multicolumn{3}{|c|}{ Pastagem fertilizada } & Pastagem não fertilizada \\
\hline g/hora & $16,8^{\mathrm{aA}}$ & $11,6^{\mathrm{bA}}$ & $9,3^{\mathrm{bA}}$ & $8,3^{\mathrm{bA}}$ \\
\hline g/dia & $403^{\mathrm{aA}}$ & $278^{\mathrm{bA}}$ & $222^{\mathrm{bA}}$ & $198^{\mathrm{bA}}$ \\
\hline Estimativa (kg/ano) & $147^{\mathrm{aA}}$ & $101^{\mathrm{bA}}$ & $81^{\mathrm{bA}}$ & $72^{\mathrm{bA}}$ \\
\hline g/dia/kg de peso vivo & $0,71^{\mathrm{aA}}$ & $0,46^{\mathrm{bA}}$ & $0,45^{\mathrm{bA}}$ & $0,43^{\mathrm{bA}}$ \\
\hline \multirow{3}{*}{$\begin{array}{l}\text { Grupo genético e categoria } \\
\text { animal }\end{array}$} & \multicolumn{4}{|c|}{ Mestiço Holandês x Zebu } \\
\hline & Vaca em lactação & Vaca não lactante & Novilha & Novilha \\
\hline & \multicolumn{3}{|c|}{ Pastagem fertilizada } & Pastagem não fertilizada \\
\hline g/hora & $13,8^{\mathrm{aA}}$ & $12,3^{\mathrm{abA}}$ & $9,5^{\mathrm{bcA}}$ & $7,6^{\mathrm{cA}}$ \\
\hline g/dia & $331^{\mathrm{aA}}$ & $295^{\mathrm{abA}}$ & $227^{\mathrm{bcA}}$ & $181^{\mathrm{cA}}$ \\
\hline Estimativa (kg/ano) & $121^{\mathrm{aA}}$ & $107^{\mathrm{abA}}$ & $83^{\mathrm{bcA}}$ & $66^{\mathrm{cA}}$ \\
\hline $\mathrm{g} / \mathrm{dia} / \mathrm{kg}$ de peso vivo & $0,79^{\mathrm{aA}}$ & $0,62^{\mathrm{aA}}$ & $0,62^{\mathrm{aA}}$ & $0,48^{\mathrm{aA}}$ \\
\hline
\end{tabular}

Letras minúsculas diferem na mesma linha $(\mathrm{p}<0,05)$. Letras maiúsculas diferem entre raças dentro da mesma categoria $(\mathrm{p}<0,05)$. Adaptado de Primavesi et al. (2004). 
Tabela 3. Produção de metano e ingestão em função da estação do ano, raça, categoria animal e sistema de produção

\begin{tabular}{|c|c|c|c|c|c|c|c|c|}
\hline \multirow{2}{*}{ Tratamento } & \multicolumn{2}{|c|}{$\mathrm{CH}_{4}$} & \multirow{2}{*}{$\begin{array}{l}\text { IMS } \\
\text { g/d }\end{array}$} & \multirow{2}{*}{$\begin{array}{c}\text { IMO } \\
\mathrm{g} / \mathrm{d}\end{array}$} & \multirow{2}{*}{$\begin{array}{c}\text { IMOD } \\
\text { g/d }\end{array}$} & \multicolumn{3}{|c|}{$\mathrm{CH}_{4}(\mathrm{~g} / \mathrm{kg})$} \\
\hline & $\mathrm{g} / \mathrm{d}$ & $\mathrm{g} / \mathrm{kg}^{0.75} \mathrm{LW}$ & & & & IMS & IMO & IMOD \\
\hline & \multicolumn{8}{|c|}{ Verão } \\
\hline & \multicolumn{8}{|c|}{ Holandesa } \\
\hline VL & 403,2 & 3.45 & 19.19 & 17.48 & 10.87 & 21.0 & 23.1 & 37.4 \\
\hline VS & 280,0 & 2.30 & 14.99 & 13.58 & 7.89 & 18.8 & 20.7 & 35.7 \\
\hline Nint & 222,2 & 2.11 & 12.78 & 11.60 & 6.81 & 17.4 & 19.2 & 32.9 \\
\hline \multirow[t]{2}{*}{ Next } & 198,4 & 1.99 & 12.21 & 11.24 & 5.61 & 16.1 & 17.5 & 36.1 \\
\hline & \multicolumn{8}{|c|}{ Mestiça } \\
\hline VL & 332,6 & 3.57 & 13.66 & 12.56 & 6.59 & 22.0 & 23.0 & 36.6 \\
\hline VS & 294,5 & 2.90 & 12.55 & 11.39 & 6.62 & 16.9 & 18.1 & 32.2 \\
\hline Nint & 232,6 & 2.75 & 10.02 & 9.12 & 5.42 & 18.7 & 20.1 & 35.3 \\
\hline \multirow[t]{3}{*}{ Next } & 181,0 & 2.12 & 10.20 & 9.39 & 4.69 & 13.5 & 14.5 & 39.5 \\
\hline & \multicolumn{8}{|c|}{ Outono } \\
\hline & \multicolumn{8}{|c|}{ Holandesa } \\
\hline VL & 383,2 & 3,28 & 17,64 & 16,86 & 10,64 & 24,4 & 26,5 & 51,0 \\
\hline VS & 261,3 & 2,04 & 15,42 & 14,36 & 8,10 & 23,8 & 26,2 & 44,7 \\
\hline Nint & 245,0 & 2,25 & 13,13 & 12,23 & 6,90 & 22,9 & 25,2 & 42,4 \\
\hline \multirow[t]{2}{*}{ Next } & 157,2 & 1,65 & 11,59 & 10,79 & 4,04 & 17,6 & 19,2 & 38,7 \\
\hline & \multicolumn{8}{|c|}{ Mestiça } \\
\hline VL & 296,3 & 2,92 & 11,81 & 10,94 & 6,37 & 25,6 & 27,6 & 47,4 \\
\hline VS & 238,4 & 2,21 & 13,56 & 12,62 & 7,10 & 17,8 & 19,1 & 33,8 \\
\hline Nint & 190,6 & 2,12 & 10,87 & 10,13 & 5,76 & 17,4 & 18,6 & 32,6 \\
\hline \multirow[t]{2}{*}{ Next } & 180,3 & 2,05 & 10,74 & 10,00 & 3,73 & 16,7 & 18,0 & 48,8 \\
\hline & \multicolumn{8}{|c|}{ Média de raça, intensivo } \\
\hline Holandesa & $299,3^{\mathrm{a}}$ & 2,74 & $15,52^{\mathrm{a}}$ & $14,4^{\mathrm{a}}$ & $8,53^{\mathrm{a}}$ & 19,1 & 20,7 & 35,0 \\
\hline Mestiças & $264,2^{\mathrm{b}}$ & 2,57 & $12,08^{\mathrm{b}}$ & $11,1^{\mathrm{b}}$ & $6,31^{\mathrm{b}}$ & 22,0 & 23,9 & 42,0 \\
\hline \multirow[t]{2}{*}{ DMS } & 28,6 & 0,23 & 0,88 & 0,84 & 0,54 & 1,8 & 1,9 & 3,2 \\
\hline & \multicolumn{8}{|c|}{ Média de categoria animal, intensivo } \\
\hline VL & $353,8^{\mathrm{a}}$ & $3,30^{\mathrm{a}}$ & $15,57^{\mathrm{a}}$ & $14,46^{\mathrm{a}}$ & $8,61^{\mathrm{a}}$ & 23,2 & 25,0 & 43,1 \\
\hline VS & $268,8^{\mathrm{b}}$ & $2,36^{\mathrm{b}}$ & $14,13^{\mathrm{b}}$ & $12,99^{\mathrm{b}}$ & $7,43^{\mathrm{b}}$ & 19,3 & 21,0 & 36,6 \\
\hline Nint & $222,6^{\mathrm{c}}$ & $2,31^{\mathrm{b}}$ & $11,70^{\mathrm{c}}$ & $10,77^{\mathrm{c}}$ & $6,22^{\mathrm{c}}$ & 19,1 & 20,8 & 35,8 \\
\hline \multirow[t]{2}{*}{ MSD } & 35,0 & 0,28 & 1,08 & 1,03 & 0,66 & 2,2 & 2,4 & 3,9 \\
\hline & & & Média & estação & ano, inter & & & \\
\hline Verão & 294,3 & $2,85^{\mathrm{a}}$ & 13,86 & 12,85 & 7,48 & 23,4 & 23,5 & 40,7 \\
\hline Outono & 269,2 & $2,47^{\mathrm{b}}$ & 13,74 & 12,62 & 7,37 & 19,7 & 21,1 & 36,3 \\
\hline DMS & 28,6 & 0,23 & 0,88 & 0,84 & 0,54 & 1,8 & 1,9 & 3,2 \\
\hline & & & Méd & e sistem & e produç & & & \\
\hline Nint & $222,6^{\mathrm{a}}$ & $2,31^{\mathrm{a}}$ & 11,70 & 10,77 & $6,22^{\mathrm{a}}$ & 19,1 & 20,8 & 35,8 \\
\hline Next & $179,2^{\mathrm{b}}$ & $1,95^{\mathrm{b}}$ & 11,18 & 10,35 & $4,52^{\mathrm{b}}$ & 16,0 & 17,3 & 40,8 \\
\hline DMS & 38,6 & 0,35 & 0,61 & 0,56 & 0,35 & 2,8 & 3,0 & 6,7 \\
\hline & & & Mé & de raça & sistema & & & \\
\hline Holandesa & 205,7 & 2,26 & $12,43^{\mathrm{a}}$ & $11,46^{\mathrm{a}}$ & $5,84^{\mathrm{a}}$ & 16,4 & 17,8 & 36,0 \\
\hline Mestiça & 196,1 & 2,00 & $10,46^{\mathrm{b}}$ & $9,66^{\mathrm{b}}$ & $4,90^{\mathrm{b}}$ & 18,7 & 20,2 & 40,6 \\
\hline DMS & 38,7 & 0,35 & 0,61 & 0,56 & 0,35 & 2,8 & 3,0 & 6,7 \\
\hline & & & Média de & tações d & no nos si & nas & & \\
\hline Verão & 208,5 & 2,24 & 11,58 & 10,79 & $5,63^{\mathrm{a}}$ & 18,5 & 20,3 & 37,5 \\
\hline Outono & 193,3 & 2,02 & 11,30 & 10,34 & $5,11^{\mathrm{b}}$ & 16,6 & 17,8 & 39,0 \\
\hline DMS & 38,6 & 0,35 & 0,61 & 0,56 & 0,35 & 2,8 & 3,0 & 6,7 \\
\hline
\end{tabular}

Médias seguidas de letras diferentes na coluna diferem estatisticamente de acordo com o teste de Tukey. DMS = Diferença mínima significativa, $\mathrm{CH}_{4}=$ Produção de metano, $\mathrm{IMS}=$ ingestão de material seca, IMO = ingestão de matéria orgânica, IMOD = ingestão de matéria orgânica digestível. VL and VS = Vacas em lactação e secas, Nint or Next $=$ Novilhas em sistema intensivo ou extensivo. Pedreira et al. (2009). 
Segundo Cottle et al. (2011), nos sistemas de produção de bovinos em pastagens, o nível de consumo, qualidade da forragem disponível e a digestibilidade da massa ingerida são fatores determinantes para a produção de metano entérico pelos animais e variam conforme a espécie forrageira, o sistema de manejo adotado e a estação do ano. Técnicas nutricionais como o uso de ionóforos, glicerol, tanino, saponinas, óleos, gorduras, vacinas, anticorpos policlonais, técnicas de manejo de pastagens, melhoramento genético e sistemas eficientes de produção têm sido utilizados para manipular o rúmen e reduzir a emissão do gás (MOHAMMED et al., 2004). Entretanto, mais pesquisas são necessárias para o entendimento da produção de metano entérico e eficiência energética de animais suplementados que são mantidos em pastagens tropicais.

A utilização de ionóforos em dietas para bovinos foi proposta por Guan et al. (2006) como uma estratégia para a mitigação das emissões de $\mathrm{CH}_{4}$ entérica. Foram avaliados os efeitos de curto e longo prazo de uma alimentação de um único ionóforo (monensina) ou rotação de dois ionóforos (Monensina e Lasalocida) nas emissões de $\mathrm{CH}_{4}$ entérico em novilhos.

$\mathrm{O}$ uso de ionóforos diminuiu $(\mathrm{P}<0,05)$ as emissões de $\mathrm{CH}_{4}$ entérico, expresso como litros por quilograma de matéria seca ingerida ou em porcentagem de energia consumida. Bovinos alimentados com rotação de ionóforos não apresentaram menor emissão, nem tiveram um período maior de redução nas emissões de $\mathrm{CH}_{4}$ entérica em comparação com bovinos que receberam somente monensina. A utilização de monensina e da rotação da monensina e lasalocida diminuiu $(\mathrm{P}<0,001)$ a população total de protozoários ciliados. Esses dados sugerem que os efeitos dos ionóforos sobre a produção de $\mathrm{CH}_{4}$ entéricos estão relacionados à populações de protozoários ciliados.

$\mathrm{O}$ uso de ionóforos pode resultar em resistência dos micro-organismos ruminais e, como resultado, o efeito é de curta duração. $\mathrm{O}$ custo elevado dos ácidos orgânicos torna-os economicamente inviáveis, se adicionados à dieta de ruminantes. No entanto, os ácidos orgânicos estão presentes em concentrações relativamente elevadas no tecido foliar das plantas, e devem ser feitas tentativas para selecionar e produzir forragens com altos níveis desses compostos.

Argumenta-se que uma estratégia mais eficiente para redução entérica de $\mathrm{CH}_{4}$ em ruminantes deve ser feita com a escolha de forragens de alta concentração de carboidratos solúveis, com leguminosas compostas de metabólitos secundários, como taninos. Frutos ou plantas que contenham saponinas também podem ser utilizados, desde que estes não afetem o consumo e a digestibilidade. $\mathrm{O}$ aperfeiçoamento da nutrição de bovinos, por meio da alimentação com forrageiras de alta qualidade, pode resultar em bom desempenho dos animais e na redução da emissão de $\mathrm{CH}_{4}$ por unidade de consumo de matéria seca e por unidade de produto (LASCANO \& CÁRDENAS, 2010).

A presença de taninos na dieta de animais está relacionada a efeitos adversos, como fatores antinutricionais, que causam menor consumo de matéria seca e reduz a digestão de proteínas e fibras. No entanto, recentemente, um efeito benéfico está associado com taninos: a redução da emissão de metano ruminal (WOODWARD et al., 2001; MAKKAR, 2003).

Oliveira et al. (2007) avaliaram o efeito de dietas que continham silagens de sorgo com altas (HT) e baixas (LT) concentrações suplementadas com 
concentrado ou ureia sobre emissão de metano ruminal em bovinos de corte. Os autores não observaram efeito dos níveis de tanino sobre a emissão de metano, entretanto, a suplementação com concentrado na dieta LT diminuiu as perdas de gás como uma função do consumo bruto de energia em comparação com a suplementação da dieta com ureia. Dessa forma, esses resultados sugerem o potencial da suplementação concentrada em minimizar as perdas de energia, como metano em ruminantes, e aumentar a eficiência de utilização de energia. A inclusão de concentrado na dieta de bovinos de corte aumentou a eficiência de utilização de energia, o que refletiu em menores perdas de metano em função da ingestão de energia bruta.

Reduzir a produção de metano entérico pode ser um benefício econômico direto, pois coincide com uma maior eficiência no uso da energia do alimento pelo animal e redução na produção de gases de efeito estufa. Adição de gorduras na dieta pode reduzir as emissões de metano (JOHNSON \& JOHNSON, 1995). Estudos atuais mostram que a adição de altos níveis (> 4\%) de gordura na suplementação para rações animais podem causar substancial redução nas emissões de metano (ou seja, > 20\%) (DOHME et al., 2001; MCGINN et al., 2004; WEISS \& PINOS-RODRIGUEZ, 2009).

A redução de $\mathrm{CO}_{2}$ e metano no rúmen desempenham importante função, visto agirem na remoção contínua de $\mathrm{H}_{2}$ resultante da fermentação de matéria orgânica. Dessa forma, a redução ou eliminação da metanogênese pode exigir o estabelecimento de outra rota para evitar o acúmulo de $\mathrm{H}_{2}$ e $\mathrm{o}$ adequado funcionamento do rúmen (WEIMER, 1998).
Uma alternativa para essa redução é o fornecimento de lipídios insaturados, por exercerem ação deletéria sobre as metanogênicas e protozoários e consumirem $\mathrm{H}_{2}$ pelo processo de biohidrogenação (MACMÜLLER et al., 1998, WADA et al., 2008). Deve-se ressaltar que a intensidade com que ocorre a inibição da produção de metano é determinada pelo grau de saturação da gordura e a quantidade suplementada (FIEVEZ et al., 2003). Nesse sentido, Onetti et al. (2001) relataram que esse comportamento do lipídio no rúmen é dependente da quantidade, do perfil de ácido graxo que compõe a fonte de lipídio, bem como da interação fonte versus ingredientes da dieta basal.

No estudo da influência de fontes lipídicas no consumo, digestibilidade, produção de metano e desempenho de bovinos de corte em pastagem, Valente e Carvalho (dados não publicados) observaram que a adição de fontes lipídicas na dieta de bovinos não reduziu a emissão de $\mathrm{CH}_{4}$ por $\mathrm{kg}$ de produto (Tabela 4). O mesmo foi observado por Fiorentini (dados não publicados) na avaliação das mesmas fontes lipídicas em animais confinados (Tabela 5).

De forma complementar às mudanças no sistema de produção, têm sido avaliadas alternativas para reduzir a produção de metano ruminal pelo animal. McCallister \& Newbold (2008) apontam três meios para reduzir o processo de metanogênese no rúmen, a saber: inibição direta da metanogênese, com o redirecionamento do $\mathrm{H}^{+}$para produtos alternativos; diminuição de produção de $\mathrm{H}^{+}$; e promoção de outros produtos que consomem $\mathrm{H}^{+}$. Esses autores comentam que diversas pesquisas a respeito da produção ruminal de metano e alternativas de redução deste mostraram que os 
diversos métodos de defaunação é eficiente por curto períodos de tempo, pois, em poucos dias, a ecologia ruminal se estabiliza e o nível de produção de metano volta ao normal.

Outra estratégia de redução de metano investigada é o desenvolvimento de uma vacina que estimularia o sistema imunológico do ruminante para produzir anticorpos contra metanogênicas (WRIGHT et al., 2004). Esses autores, em um estudo realizado com ovinos, observaram que o uso das vacinas reduziu em $7,7 \%$ a produção de metano, quando duas vacinas, a VF3 (baseada em três cepas metanogênicas) e VF7 (baseada em sete amostras metanogênicas), foram testadas.

Tabela 4. Ganho médio diário e emissão de metano de novilhos Nelore suplementados em pastagem de capim Xaraés com diferentes fontes de lipídios durante a recria $(0,5 \% \mathrm{PC})$ e, terminação $(1,0 \% \mathrm{PC})$

\begin{tabular}{|c|c|c|c|c|c|c|}
\hline \multirow[b]{2}{*}{ Variáveis } & \multicolumn{5}{|c|}{ Fontes de lipídios } & \multirow[b]{2}{*}{$\mathrm{CV}(\%)$} \\
\hline & Controle & $\begin{array}{l}\text { Óleo de } \\
\text { palma }\end{array}$ & $\begin{array}{l}\text { Óleo de } \\
\text { linhaça }\end{array}$ & $\begin{array}{c}\text { Gordura } \\
\text { protegida }\end{array}$ & $\begin{array}{l}\text { Soja } \\
\text { grão }\end{array}$ & \\
\hline \multicolumn{7}{|c|}{ Recria } \\
\hline GMD & $0,724^{\mathrm{b}}$ & $0,697^{\mathrm{b}}$ & $0,759^{\mathrm{ab}}$ & $0,864^{\mathrm{a}}$ & $0,780^{\mathrm{ab}}$ & 17,2 \\
\hline $\mathrm{g} \mathrm{CH}_{4} / \mathrm{dia}$ & 189,56 & 167,40 & 215,28 & 164,73 & 179,82 & 37,0 \\
\hline $\mathrm{mg} \mathrm{CH}_{4} / \mathrm{dia} / \mathrm{PC}$ & 447,56 & 443,47 & 553,25 & 376,34 & 410,93 & 41,5 \\
\hline \multicolumn{7}{|c|}{ Terminação } \\
\hline GMD & 0,56 & 0,57 & 0,66 & 0,56 & 0,56 & 12,1 \\
\hline $\mathrm{g} \mathrm{CH}_{4} / \mathrm{dia}$ & 113,73 & 112,64 & 70,18 & 101,88 & 82,47 & 19,4 \\
\hline $\mathrm{mg} \mathrm{CH}_{4} / \mathrm{dia} / \mathrm{PC}$ & 238.65 & 228,51 & 147,04 & 208,54 & 180,84 & 11,8 \\
\hline $\begin{array}{l}\mathrm{kg} \mathrm{CH}_{4} / \mathrm{kg} \text { de } \\
\text { produto }\end{array}$ & 0,24 & 0,24 & 0,14 & 0,23 & 0,18 & 22,6 \\
\hline
\end{tabular}

GMD = ganho médio diário; $\mathrm{PC}=$ peso corporal.

Médias seguidas de letras minúsculas diferentes, na mesma linha, diferem pelo teste de Tukey a 5\%. Dados não publicados (Valente e Carvalho).

Tabela 5. Emissão de metano de novilhos Nelore em confinamento que recebiam diferentes fontes de lipídios

\begin{tabular}{|c|c|c|c|c|c|c|c|}
\hline \multirow[b]{2}{*}{ Variável } & \multicolumn{5}{|c|}{ Fontes de lipídios $^{1}$} & \multirow{2}{*}{$\begin{array}{l}\mathrm{CV} \\
(\%)\end{array}$} & \multirow[b]{2}{*}{$\mathrm{P}$} \\
\hline & Controle & $\begin{array}{c}\text { Óleo de } \\
\text { palma }\end{array}$ & $\begin{array}{l}\text { Óleo de } \\
\text { linhaça }\end{array}$ & $\begin{array}{c}\text { Gordura } \\
\text { protegida }\end{array}$ & $\begin{array}{l}\text { Soja } \\
\text { grão }\end{array}$ & & \\
\hline $\mathrm{g} \mathrm{CH}_{4} / \mathrm{dia}$ & $147,25^{\mathrm{a}}$ & $66,70^{\mathrm{b}}$ & $62,77^{\mathrm{b}}$ & $110,65^{\mathrm{ab}}$ & $63,98^{\mathrm{b}}$ & 24,8 & 0,0005 \\
\hline $\mathrm{mg} \mathrm{CH}_{4} / \mathrm{d} / \mathrm{kg} \mathrm{PC}$ & $296,83^{\mathrm{a}}$ & $147,92^{\mathrm{b}}$ & $136,98^{\mathrm{b}}$ & $231,41^{\mathrm{ab}}$ & $143,97^{\mathrm{b}}$ & 23,0 & 0,0008 \\
\hline $\begin{array}{l}\mathrm{kg} \text { de } \mathrm{CH}_{4} / \mathrm{kg} \text { de } \\
\text { produto }\end{array}$ & 0,22 & 0,29 & 0,16 & 0,20 & 0,17 & 37,3 & 0,2458 \\
\hline
\end{tabular}

${ }^{\mathrm{T}}$ Dieta composta de $60 \%$ de silagem de milho e $40 \%$ de concentrado.

$\mathrm{CV}=$ coeficiente de variação (\%); Probabilidade; Médias seguidas de letras minúsculas diferentes, na mesma linha, diferem pelo teste de Tukey a 5\%. Dados não publicados (Fiorentini). 
Willians et al. (2009) estudaram uma vacina baseada em cinco linhagens metanogênicas, administrada em três doses para ovinos, e observaram que a produção de metano aumentou $18 \%$ com a vacinação, o que os levou a acreditar que a vacina não abrangeu a segmentação dos micro-organismos metanogênicos capazes de produzir a maioria do metano. No entanto, Wright et al. (2007) afirmaram que mesmo que alguma vacina seja encontrada, esta é específica para cada situação, pois a população metanogênica presente pode variar com base na dieta e localização geográfica do hospedeiro, de modo a aumentar o desafio de se trabalhar com imunizadores.

Outro ponto que tem que ser considerado é o balanço entre a emissão de $\mathrm{CO}_{2}$ pelos animais e a absorção deste pelas gramíneas e forrageiras das pastagens. O potencial de mitigação do sequestro de carbono do solo, em sistemas de pastagem, pode ser significativamente superior que emissões de metano resultantes da fermentação entérica ou manejo de dejetos.

Os 3,4 bilhões de hectares de pastagens do mundo também podem desempenhar um importante papel no apoio à adaptação e redução da vulnerabilidade às mudanças climáticas de mais de um bilhão de pessoas que dependem da pecuária como meio de subsistência (FAO, 2009). Melhores práticas de manejo que restabelecem a matéria orgânica aos solos de pastagens reduzem a erosão e diminuem perdas com queima da vegetação e sobrecarga (superpastejo) podem, portanto, ajudar a sequestrar grandes quantidades de carbono, até um bilhão de toneladas por ano, segundo algumas estimativas. Contudo, isso exigiria um esforço global intenso e coordenado, além de financiamento adequado e, principalmente, a superação de barreiras sociopolíticas e econômicas.

Ao se considerar a importância dos ruminantes para economia mundial, é essencial estabelecer, sustentavelmente, formas de aumento da produtividade que procurem reduzir a emissão de metano, a qual tem sido pauta dos sistemas de comunicação.

Vale relembrar a importância da divulgação das pesquisas na área que mostram inovações tecnológicas e conhecimentos a serem gerados para avaliar as principais fontes de emissão de gases do efeito estufa e mitigar sua expulsão: metano $\left(\mathrm{CH}_{4}\right)$, óxido nitroso $\left(\mathrm{N}_{2} \mathrm{O}\right)$ e dióxido de carbono $\left(\mathrm{CO}_{2}\right)$. Essa mitigação visa obter a sustentabilidade da pastagem e o balanço positivo desses gases na atividade pecuária para atender à exigência de colocá-la na vertente da sustentabilidade.

\section{REFERÊNCIAS}

BARIONI, L.G.; LIMA, M.A.; ZEN, S.D.; GUIMARÃES JÚNIOR, R.; FERREIRA, A.C. A baseline projection of methane emissions by the Brazilian beef sector: preliminary results. In: GREENHOUSES GAS AND ANIMAL AGRICULTURE CONFERENCE, 2007 Christchurch, NZ. Proceedings... Christchurch, NZ. 2007.

BEAUCHEMIN, K.A.; KREUZER, M.; O'MARAC, F.; McALLISTER, T.A. Nutritional management for enteric methane abatement: a review.

Australian Journal of Experimental Agriculture, v.48, p.21-27, 2008. 
BRASIL. Ministério da Ciência e Tecnologia. Comunicação Nacional Inicial do Brasil à Convenção-Quadro das Nações Unidas sobre Mudança do Clima. Coordenação-Geral de Mudanças Globais de Clima. Brasília, 2004. 74p. (Comunicação Nacional).

CANESIN, R.C. Frequência da suplementação de bovinos da raça Nelore mantidos em pastagens. 2009. 119f. Tese (Doutorado em Zootecnia) Universidade Estadual Paulista, Faculdade de Ciências Agrárias e Veterinárias, Jaboticabal.

COTTLE, D.J.; NOLAN, J.V.; WIEDEMANN, S.G. Ruminant enteric methane mitigation : a review. Animal Production Science, v.51, p.491-514, 2011.

DOHME, F.; MACHMÜLLER, A.; WASSERFALLEN, A.; KREUZER, M. Ruminal methanogenesis as influenced by individual fatty acids supplemented to complete ruminant diets. Letters in Applied Microbiology, v.32, p.47- 51, 2001.

FOOD AND AGRICULTURE ORGANIZATION OF THE UNITED NATIONS - FAO. Review of evidence on drylands pastoral systems and climate change. Rome, Italy. 2009.

FELIICIO, P.E. Boas e más notícias deste início de ano. 2008. Disponível: <http://www.abcz.org.br/artigos/>. Acesso em: 16 mar 2011.

FIEVEZ, V.; DOHME, F.; DANNEELS, M,; RAES, K.; DEMEYER, D. Fish oils as potent rumen methane inhibitors and associated effects on rumen fermentation in vitro and in vivo. Animal Feed Science and Technology, v.104, p.41-58, 2003.
GUAN, H.; WITTENBERG, K.M.; OMINSKI, K.H.; KRAUSE, D.O. Efficacy of ionophores in cattle diets for mitigation of enteric methane. Journal of Animal Science, v.84, p.1896-1906, 2006.

HOOK, S.E.; WRIGHT A-D.G.; MCBRIDE B.W. Methanogens: methane producers of the rumen and mitigation strategies. Archaea, v.2010, p.1-11, 2010.

INSTITUTO BRASILEIRO DE GEOGRAFIA E ESTATÍSTICA IBGE. Censo Agropecuário. 2010. Disponível em:<http://www.ibge.com.br/home/pr esidencia/noticias/noticia_visualiza.ph p?id_noticia $=1761 \& i d \_p a g i n a=1>$. Acesso em: 16 mar 2011.

INTERGOVERNMENTAL PANEL ON CLIMATE CHANGE - IPCC.

Revised IPCC guidelines for national greenhouse gas inventories. Cambridge: University Press, 2006. $297 p$.

JOHNSON, K.A.; JOHNSON, D.E. Methane emissions from Cattle. Journal of Animal Science, v.73, p.2483-2492, 1995.

KURIHARA, M.; MAGNER, T.; HUNTER, R.A.; MCCRABB, G.J. Methane production and energy partition of cattle in the tropics. British Journal of Nutrition, v.81, p.227, 1999.

LASCANO, C.E.; CÁRDENAS, E. Alternatives for methane emission mitigation in livestock systems. Revista Brasileira de Zootecnia, v.39, p.175-182, 2010. Supl. especial. 
MACHMÜLLER, A.; OSSOWSKI, D.A.; WANNER, M.; KREUSER, M. Potencial of various fatty feeds to reduce methane release from rumen fermentation in vitro (Rusitec). Animal Feed Science and Technology, v.2, p.117-130, 1998.

MAKKAR, H.P.S. Effect and fate of tannins in ruminant animals, adaptation to tannins, and strategies to overcome detrimental effects of feeding tanninrich feeds. Small Ruminant Research, v.49, p.241-256, 2003.

McCALLISTER, T.A.; NEWBOLD, C.J. Redirecting rumen fermentation to reduce methanogenesis. Australian Journal of Experimental Agriculture, v.48, p.7-13, 2008.

MCGINN, S.M.; BEAUCHEMIN, K.A.; COATES, T.; COLOMBATTO, D. Methane emissions from beef cattle: Effects of monensin, sunflower oil, enzymes, yeast, and furmaric acid. Journal of Animal Science, v. 82, p.3346-3356, 2004.

MINISTÉRIO DA CIÊNCIA E TECNOLOGIA - MCT. Relatório de referência para o Inventário nacional de emissão de gases de efeito estufa por atividades agrícolas: emissão de metano proveniente da pecuária no Brasil. Brasília, 2010.

MOHAMMED, N.; ONODERA, R.; ITABASHI, H.; LILA, Z.A. Effects of ionophores, vitamin $\mathrm{B} 6$ and distiller's grains on in vitro tryptophan biosynthesis from indolepyruvic acid, and production of other related compounds by ruminal bacteria and protozoa. Animal Feed Science and Technology, v.116, p.301-311, 2004.
NELSON, C.J.; MOSER, L.E. Plant factors affecting forage quality. In: FAHEY JUNIOR, G.C. (Ed). Forage quality, evaluation and utilization. Madison: American Society of Agronomy, 1994. p.115-154.

OLIVEIRA, S.G.; BERCHIELLI, T.T. PEDREIRA, M.S.; PRIMAVESI, O.; FRIGHETTO, R.T.; LIMA, M.A. Effect of tannin levels in sorghum silage and concentrate supplementation on apparent digestibility and methane emission in beef cattle. Animal Feed Science and Technology, v.135, p.236-248, 2007.

ONETTI, S.G.; SHAVER, R.D.; MCGUIRE, M.A.; GRUMMER, R.R. Effect of type and level of dietary fat on rumen fermentation and performance of dairy cows fed corn silage based diets. Journal of Dairy Science, v.84, p.27512759, 2001.

PEDREIRA, M.S.; PRIMAVESI, O.; LIMA, M.A.; FRIGHETTO, R.T.;.OLIVEIRA, S.G.; BERCHIELLI, T.T. Ruminal methane emission by dairy cattle in southeast Brazil. Scientia Agricola, v.66, p.742-750, 2009.

PINEDA, N. O metano e o boi brasileiro. Revista DBO, p.84-85, 2010.

PRIMAVESI, O.; FRIGHETTO, R.T.; PEDREIRA, M.S.; LIMA, M.A.; BERCHIELLI, T.T.; BARBOSA, P.F. Metano entérico de bovinos leiteiros em condições tropicais brasileiras. Pesquisa Agropecuária Brasileira, v.39, p.277283, 2004.

RIVERA, A.R.; BERCHIELLI, T.T.; MESSANA, J.D.; VELASQUEZ, P.T.; FRANCO, A.V.M.; FERNANDES, L.B. Fermentação ruminal e produção de metano em bovinos alimentados com feno de capim-tifton 85 e concentrado com aditivos. Revista Brasileira de Zootecnia, v.39, p.617-624, 2010. 
SHIBATA, M; TERADA, F. Factors affecting methane production and mitigation in ruminants. In: Review article. Animal Science Journal, v.81, p.2-10, 2010.

\section{UNITED STATES}

\section{ENVIRONMENTAL PROTECTION}

AGENCY - USEPA. Evaluating ruminant livestock efficiency projects and programs. Washington, D.C.: Peer Review Draft, 2000. 48p.

WADA, F.Y.; PRADO, IN.; SILVA, R.R.; MOLETTA, J.L.; VISENTAINER, J.V; ZEOULA, L.M. Grãos de linhaça e canola sobre o desempenho, digestibilidade aparente e característica de carcaça de novilhas nelore terminada em confinamento. Ciência Animal Brasileira, v.9, p.883895, 2008.

WEIMER, P.J. Manipulating ruminal fermentation: a microbial ecological perspective. Journal of Animal Science, v.76, p.3114-3122, 1998.

WEISS, W.P.; PINOS-RODRIGUEZ, J.M. Production responses of dairy cows when fed supplemental fat in lowand high-forage diets. Journal Dairy Science, v.92, p.6144-6155, 2009.

WILLIAMS, Y.J.; POPOVSKI, S.; REA, S.M.; SKILLMAN, L.C.; TOOVEY, A.F.; NORTHWOOD, K.S.; WRIGHT, A.D.G. A vaccine against rumen methanogens can alter the composition of archaeal populations. Applied and Environmental Microbiology, v.75, p.1860-1866, 2009.
WOODWARD, S.L.; WAGHORN, G.C.; ULYATT, M.J.; LASSEY, K.R.

Early indications that feeding lotus will reduce methane emissions from ruminants. In: NEW ZEALAND SOCIETY OF ANIMAL PRODUCTION, 2001, Adelaide. Proceedings... Adelaide: ACIAR, 2001. p.23-26.

WRIGHT, A.D.G; AUCKLAND, C.H.; LYNN, D.H. Molecular diversity of methanogens in feedlot cattle from Ontario and Prince Edward Island, Canada, Applied and Environmental Microbiology, v.73, p.4206-4210, 2007.

WRIGHT, A.D.G; KENNEDY, P.; O'NEILL, C.J.; TOOVEY, A.F.; POPOVSKI, S.; REA, S.M.; PIMM, C.L.; KLEIN, L. Reducing methane emissions in sheep by immunization against rumen methanogens. Vaccine, v.22, p.3976-3985, 2004.

ZEN, S.; BARIONI, L.G.; BONATO, D.B.B.; ALMEIDA, M.H.S.P.; RITLL, T.F. 2008. Pecuária de corte brasileira: impactos ambientais e emissões de gases efeito estufa (GEE). Disponível em:

$<$ http://www.cepea.esalq.usp.br/pdf/Cep ea_Carbono_pecuaria_SumExec.pdf $>$. Acesso em:

Data de recebimento: 10/11/2011

Data de aprovação: 01/11/2012 